\title{
Prevalencia de síndrome metabólico en médicos residentes
}

\author{
Rosa María Rodríguez-Medina ${ }^{1 *}$, Ernesto Moctezuma-Cruz ${ }^{2}$ y Dora María Becerra-López ${ }^{3}$ \\ ${ }^{1}$ Universidad de Guanajuato, Campus León, Departamento de Enfermería y Obstetricia, León; 2Instituto Mexicano del Seguro Social, Unidad de Medicina Familiar n. ${ }^{5} 55$, \\ León; ${ }^{3}$ nstituto Mexicano del Seguro Social, Unidad de Medicina Familiar n. ${ }^{\circ}$ 77, San Luis Potosí. México
}

\begin{abstract}
RESUMEN
Introducción: El síndrome metabólico (SM) se define como un conjunto de alteraciones metabólicas que aumentan el riesgo de diabetes mellitus tipo 2 y enfermedad cardiovascular. Existe evidencia de que la prevalencia de SM podría ser más elevada en profesionales de la salud en formación que en la población general. El objetivo fue determinar la prevalencia de SM y sus componentes individuales en médicos residentes. Métodos: Estudio descriptivo y de sección transversal. La muestra incluyó a 56 médicos residentes, a los cuales se les midió la composición corporal, se les aplicó recordatorio de 24 horas y se les tomó muestra de sangre previo periodo de ayuno. Para el diagnóstico de SM se aplicaron los criterios de la Federación Internacional de Diabetes (IDF). Resultados: La edad media de los participantes fue de $30.7 \pm 3.4$ años; la alteración metabólica que se identificó con mayor frecuencia fue la obesidad central $(p=0.026)$ y, de acuerdo con los criterios de la IDF, la prevalencia del SM fue del $35.7 \%$, y mayor en los hombres (51.7\%). Conclusiones: La prevalencia de SM en médicos residentes fue mayor a la prevalencia nacional descrita en población general, por lo que es necesario vigilar estrechamente su estado de salud para prevenir complicaciones.
\end{abstract}

Palabras clave: Síndrome X metabólico. Obesidad. Hipertensión.

\section{ABSTRACT}

Introduction: Metabolic Syndrome (MS) is defined as a set of metabolic alterations that increase the risk of type 2 diabetes mellitus and cardiovascular disease. There is evidence that the prevalence of MS could be higher in health personnel in training than in the general population. The objective was to determine the prevalence of MS and its individual components in medical residents. Methods: Descriptive and cross-sectional study. The sample included 56 resident physicians; body composition was measured, a 24-hour reminder was applied, and blood samples were taken after a fasting period. For the diagnosis of MS, the criteria of the International Diabetes Federation (IDF) were applied. Results: The average age of the participants was $30.7 \pm 3.4$ years; the metabolic alteration that was identified with higher frequency was central obesity $(p=0.026)$ and, according to the IDF criteria, the prevalence of MS was $35.7 \%$, and higher in men (51.7\%). Conclusions: The prevalence of MS in medical residents was higher than

\section{Correspondencia:}

*Rosa María Rodríguez Medina

E-mail: anidem_2_2@ hotmail.com
Date of reception:30-10-2018

Date of acceptance: 22-03-2019

DOI: 10.24875/HMCM.19000170 
the national prevalence described in the general population, so it is necessary to closely monitor their health status to prevent complications. (Hosp Med Clin Manag. 2019;12:22-7)

Corresponding author: Rosa María Rodríguez Medina, anidem_2_2@ hotmail.com

Key words: Metabolic X syndrome. Obesity. Hypertension.

\section{INTRODUCCIÓN}

El síndrome metabólico (SM) se define como un conjunto de alteraciones metabólicas que aumentan el riesgo de desarrollar diabetes mellitus tipo 2 y enfermedad cardiovascular, como infarto agudo al miocardio, enfermedad arterial periférica y evento vascular cerebral ${ }^{1}$. Mediante un consenso internacional se han desarrollado criterios clínicos para diagnosticar el SM; los más ampliamente aceptados han sido establecidos por la Federación Internacional de Diabetes (IDF), siendo un prerrequisito la obesidad central medida por la circunferencia de cintura, además de la disminución de colesterol HDL (lipoproteínas de alta densidad), la elevación de triglicéridos, el aumento de la presión arterial y la hiperglucemia $^{2}$. Actualmente, el SM se considera un problema de salud pública prioritario debido al aumento de su prevalencia en los últimos años, la cual oscila entre el 13.6 y el $54.4 \%$ dependiendo de los criterios aplicados para su diagnóstico ${ }^{3}$. Cabe mencionar que el incremento paralelo en la prevalencia de sobrepeso y obesidad complica aún más el panorama epidemiológico de esta enfermedad, ya que de acuerdo con la Encuesta Nacional de Salud y Nutrición (ENSANUT 2016, México), la prevalencia combinada de sobrepeso y obesidad aumentó al $72.5 \%$ para las personas mayores de 20 años ${ }^{4}$.

Por otra parte, estudios previos han identificado la prevalencia de SM en población general y específicamente en el personal de salud. Al respecto, Escasany, et al. identificaron a la edad y las jornadas de trabajo nocturnas como factores relacionados con el aumento de la prevalencia de obesidad y SM en el personal de enfermería $^{5}$. En ese estudio la prevalencia de sobrepeso y obesidad fue del 35 y el $41 \%$, respectivamente. Además, se encontró una prevalencia de SM del 33.3\% y el componente que se presentó con mayor frecuencia fue la obesidad central, en el $92 \%$ de los participantes ${ }^{5}$. En otro estudio, Orozco, et al. determinaron la prevalencia de factores de riesgo cardiovascular y su asociación con la actividad laboral que desempeñaban los profesionales de salud de dos hospitales del Instituto Mexicano del Seguro Social (IMSS) 6 . La edad media de los participantes fue de $41 \pm 9$ años y el $76 \%$ fueron mujeres. Además, la prevalencia de sobrepeso/obesidad fue del $73 \%$ y la prevalencia de SM fue del $32.5 \%$; este último se asoció con el grupo de asistentes médicas ((odds ratio [OR]: 2.73; IC 95\%: 1.31-5.69) y nutrición/dietética (OR: 2.6; IC 95\%: 1.31-5.24) ${ }^{6}$.

Dado el panorama actual, el objetivo del presente estudio fue determinar la prevalencia de SM y sus componentes individuales en médicos residentes del IMSS en San Luis Potosí, México. Lo anterior permitirá establecer estrategias para fomentar estilos de vida saludables y prevenir complicaciones que deterioren su calidad de vida.

\section{MÉTODOS}

Estudio de sección transversal que incluyó a 56 médicos residentes del IMSS en San Luis Potosí, S.L.P., durante el ciclo 2014-2015. Se incluyeron aquellos residentes que cumplieron con los criterios de inclusión y aceptaron participar en el estudio previo consentimiento informado.

\section{Evaluación clínica}

A todos los participantes se les midió peso, talla, índice de masa corporal (IMC), circunferencia de cintura y presión arterial. La antropometría se realizó por personal capacitado de acuerdo con las recomendaciones de la Sociedad Internacional para el Avance de la Cineantropometría $(\mathrm{ISAK})^{7}$. La presión arterial se tomó con el esfigmomanómetro aneroide (Welch Allyn Durashock ${ }^{\mathrm{TM}}$ adult) previamente calibrado y siguiendo la técnica estandarizada por la Asociación Americana del Corazón ${ }^{8}$. Posteriormente, se les aplicó recordatorio de 24 horas para calcular la cantidad de carbohidratos, proteínas, lípidos y kilocalorías de los alimentos consumidos.

\section{Análisis metabólico}

Se tomó una muestra de sangre por punción de una vena antecubital con un sistema Vacutainer ${ }^{\circledR}$ previo ayuno de 
8 horas. Se determinaron los niveles de glucosa, colesterol total, cLDL, CHDL y triglicéridos. La glucosa se midió inmediatamente con el método enzimático colorimétrico y la técnica glucosa oxidasa/peroxidasa (BioSystems ${ }^{\circledR}$, EE.UU.). De igual forma, el colesterol y los triglicéridos se determinaron mediante métodos enzimáticos colorimétricos Colesterol oxidasa- peroxidasa (CHOD- POD) y Glicerol- 3- oxidasa- peroxidasa (GPO-POD), respectivamente (Spinreact, España). Todas las determinaciones fueron realizadas en el Laboratorio de la Unidad de Medicina Familiar n. ${ }^{\circ} 47$ de San Luis Potosí, S.L.P, siguiendo las normas de bioseguridad vigentes ${ }^{9}$.

\section{Definición de síndrome metabólico}

Para determinar la prevalencia de SM se aplicaron los criterios de la IDF, los cuales consideran la presencia de obesidad central como un criterio obligatorio, definida como una circunferencia de cintura $>90 \mathrm{~cm}$ en los hombres y $>80 \mathrm{~cm}$ en las mujeres, además de dos de los siguientes cuatro criterios: a) hipertrigliceridemia ( $\geq 150 \mathrm{mg} / \mathrm{dl}$ ) o tratamiento específico para la anormalidad lipídica; b) c-HDL bajo (< $40 \mathrm{mg} / \mathrm{dl}$ en hombres $y<50 \mathrm{mg} / \mathrm{dl}$ en mujeres) o tratamiento específico para la anormalidad lipídica; c) aumento en las cifras de presión arterial (sistólica $\geq 130 \mathrm{mmHg}$ o bien diastólica $\geq 85 \mathrm{mmHg}$ ) o tratamiento de hipertensión arterial, diagnosticada previamente, o d) glucemia plasmática en ayuno igual o mayor de $100 \mathrm{mg} / \mathrm{dl}$ o Diabetes mellitus tipo 2 (DM2) diagnosticada previamente ${ }^{10,11}$.

\section{Análisis estadístico}

Se realizó una base de datos en el programa SPSS ${ }^{\circledR}$, versión 21. Las variables cuantitativas se reportaron con media y desviación estándar; las variables cualitativas se reportaron en forma de frecuencias y porcentajes. Se realizó la prueba de bondad del ajuste de KolmogorovSmirnov con corrección de Lilliefors para determinar la normalidad en la distribución de los datos. Se examinaron las diferencias entre la composición corporal, el perfil metabólico y la ingesta de macronutrientes en los participantes con y sin SM mediante de la prueba t para muestras independientes y la prueba chi cuadrada. Se aceptó un valor de $p<0.05$ para la significancia estadística.

\section{Consideraciones éticas}

El protocolo de investigación fue aprobado por el Comité de Investigación del IMSS con el siguiente número de registro: R-2014 2402-51. Para preservar la identidad e integridad de los participantes, la información que proporcionaron se manejó confidencialmente, con respeto y únicamente para cumplir el objetivo del presente estudio. Los resultados de las valoraciones se entregaron en un sobre cerrado y de manera individual a cada participante. Se dio cumplimiento a la Ley General de Salud en materia de investigación para la salud y a la Declaración de Helsinki ${ }^{12,13}$.

\section{Resultados}

En el presente estudió participaron 56 médicos residentes de Medicina Familiar (29 hombres y 27 mujeres) con una edad media de $30.7 \pm 3.4$ años. De acuerdo con los criterios de la IDF se encontró una prevalencia del SM de $35.7 \%$, mayor en los hombres (51.7\%), en los participantes menores de 30 años (37.1\%) y en los residentes de segundo grado (44.4\%).

\section{Características de los participantes}

En la tabla 1 se presentan las características antropométricas, metabólicas y el consumo de macronutrientes de los participantes con y sin SM. Como se observa, aquellos con SM mostraron mayor IMC y circunferencia de cintura, además se observaron diferencias en los niveles de glucosa, cHDL y triglicéridos. En relación al consumo de macronutrientes, los participantes con SM mostraron mayor consumo de hidratos de carbono $(\mathrm{HCO})(p<0.001)$, proteínas $(p=0.002)$, lípidos $(p<0.001)$ y kilocalorías totales $(p<0.001)$.

En la figura 1 se presenta a los participantes con y sin SM de acuerdo con su IMC. Se observó que la mayoría de los participantes en ambos grupos presentaron sobrepeso de acuerdo a los puntos de corte de la Organización Mundial de la Salud (OMS). Cabe mencionar que ninguno de los participantes con SM presentó peso normal.

\section{Criterios de la Federación Internacional de Diabetes para el diagnóstico de síndrome metabólico}

Se determinó la frecuencia de los componentes del $\mathrm{SM}$ en ambos sexos y se observó que en los hombres el criterio que se presentó con mayor frecuencia fue la obesidad central medida por la circunferencia de cintura $(p=0.026)$. No se observaron diferencias al 
Tabla 1. Características de los participantes con y sin síndrome metabólico

\begin{tabular}{|c|c|c|c|c|}
\hline Características & $\begin{array}{l}\text { SM (+) } \\
\mathrm{n}=20\end{array}$ & $\begin{array}{l}\text { SM (-) } \\
\mathrm{n}=36\end{array}$ & Valor $\mathrm{t}$ & Valor $p$ \\
\hline Edad (años) & $31.2 \pm 4.2$ & $30.4 \pm 3.1$ & -0.825 & 0.413 \\
\hline $\begin{array}{c}\text { Sexo, n }(\%) \\
\text { Masculino } \\
\text { Femenino }\end{array}$ & $\begin{array}{c}15(75) \\
5(25)\end{array}$ & $\begin{array}{l}14(38.9) \\
22(61.1)\end{array}$ & chi cuadrada & 0.010 \\
\hline Peso (kg) & $83.6 \pm 9.8$ & $71.9 \pm 14.2$ & -3.269 & 0.002 \\
\hline Talla (m) & $1.7 \pm 0.06$ & $1.6 \pm 0.09$ & -1.792 & 0.079 \\
\hline IMC $\left(\mathrm{kg} / \mathrm{m}^{2}\right)$ & $29.6 \pm 2.8$ & $26.5 \pm 4.11$ & -2.987 & 0.004 \\
\hline Circunferencia cintura (cm) & $98.6 \pm 9.5$ & $89.6 \pm 11.2$ & -3.002 & 0.004 \\
\hline Glucosa (mg/dl) & $93.3 \pm 30.2$ & $83.1 \pm 5.6$ & -1.986 & 0.052 \\
\hline \multicolumn{5}{|l|}{ Colesterol total (mg/dl) } \\
\hline $\begin{array}{l}\mathrm{cHDL}(\mathrm{mg} / \mathrm{dl}) \\
\mathrm{cLDL}(\mathrm{mg} / \mathrm{dl})\end{array}$ & $35.5 \pm 5.5$ & $47.4 \pm 12.1$ & 4.131 & $<0.001$ \\
\hline Triglicéridos (mg/dl) & $248.7 \pm 52.2$ & $108.7 \pm 36.1$ & -5.927 & $<0.001$ \\
\hline $\mathrm{HCO}(\mathrm{g})$ & $427.9 \pm 160.2$ & $219.6 \pm 96.6$ & -6.079 & $<0.001$ \\
\hline Proteínas (g) & $165.0 \pm 73.4$ & $101 \pm 52.1$ & -3.791 & 0.002 \\
\hline Lípidos (g) & $210.5 \pm 90.6$ & $96.5 \pm 70$ & -5.245 & $<0.001$ \\
\hline Kilocalorías totales & $3,420.8 \pm 1,101.6$ & $2,127.7 \pm 827$ & -4.970 & $<0.001$ \\
\hline
\end{tabular}

Los valores son presentados con media \pm DE: Desviación estándar. Comparación a través de la prueba t de Student para muestras independientes y chi cuadrada en el caso de proporciones. SM: síndrome metabólico; IMC: índice de masa corporal; HCO: hidratos de carbono.

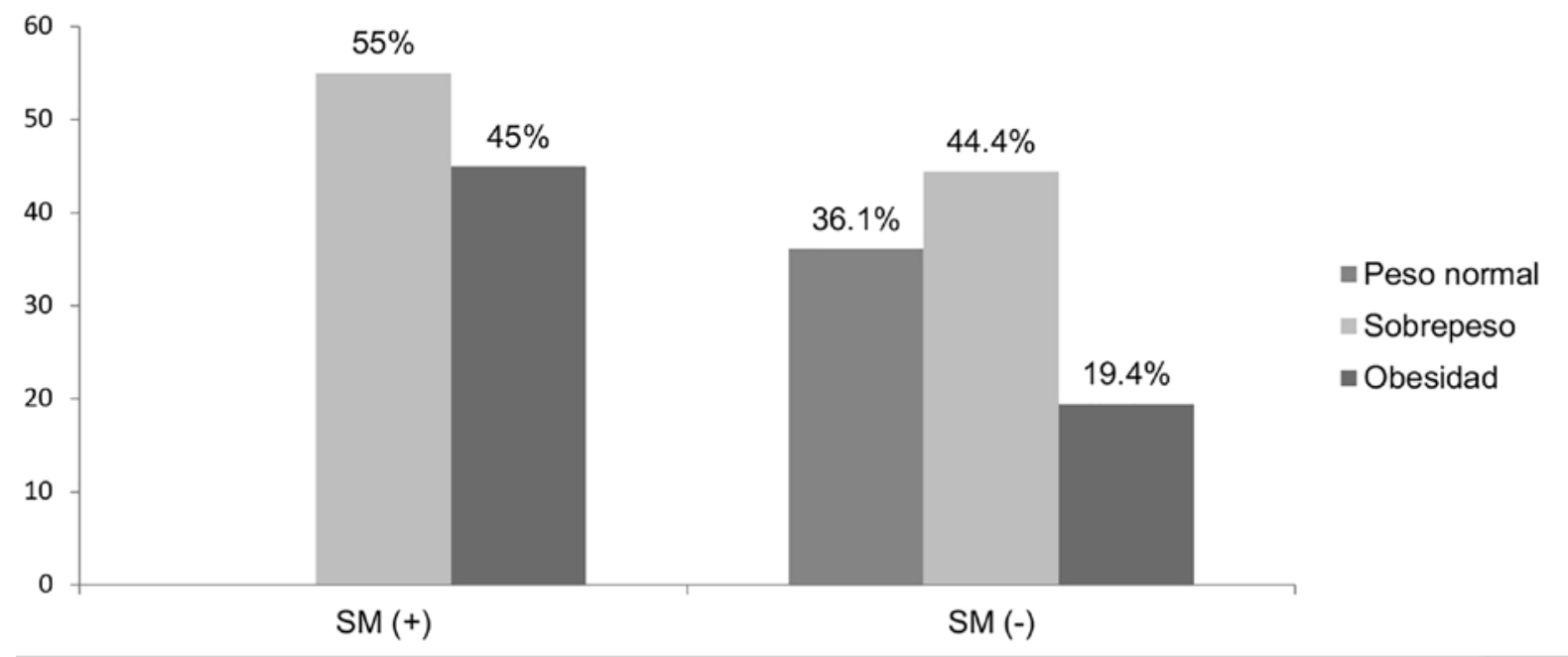

Figura 1. Participantes con y sin síndrome metabólico (SM) según su índice de masa corporal.

comparar la proporción de los otros componentes del SM en ambos sexos (Fig. 2). Por otra parte, se identificó el número de alteraciones metabólicas de acuerdo al IMC de los participantes (Fig. 3). Cabe resaltar que todos los participantes que cumplían con los cuatro criterios de la IDF para el diagnóstico de SM también presentaban obesidad de acuerdo a su IMC. En contraste, los participantes sin ningún criterio para
SM presentaron peso normal (23.1\%) y sobrepeso $(3.7 \%)$.

\section{DISCUSIÓN}

En el presente estudio se encontró una prevalencia de SM mayor a la reportada en otros países e incluso 


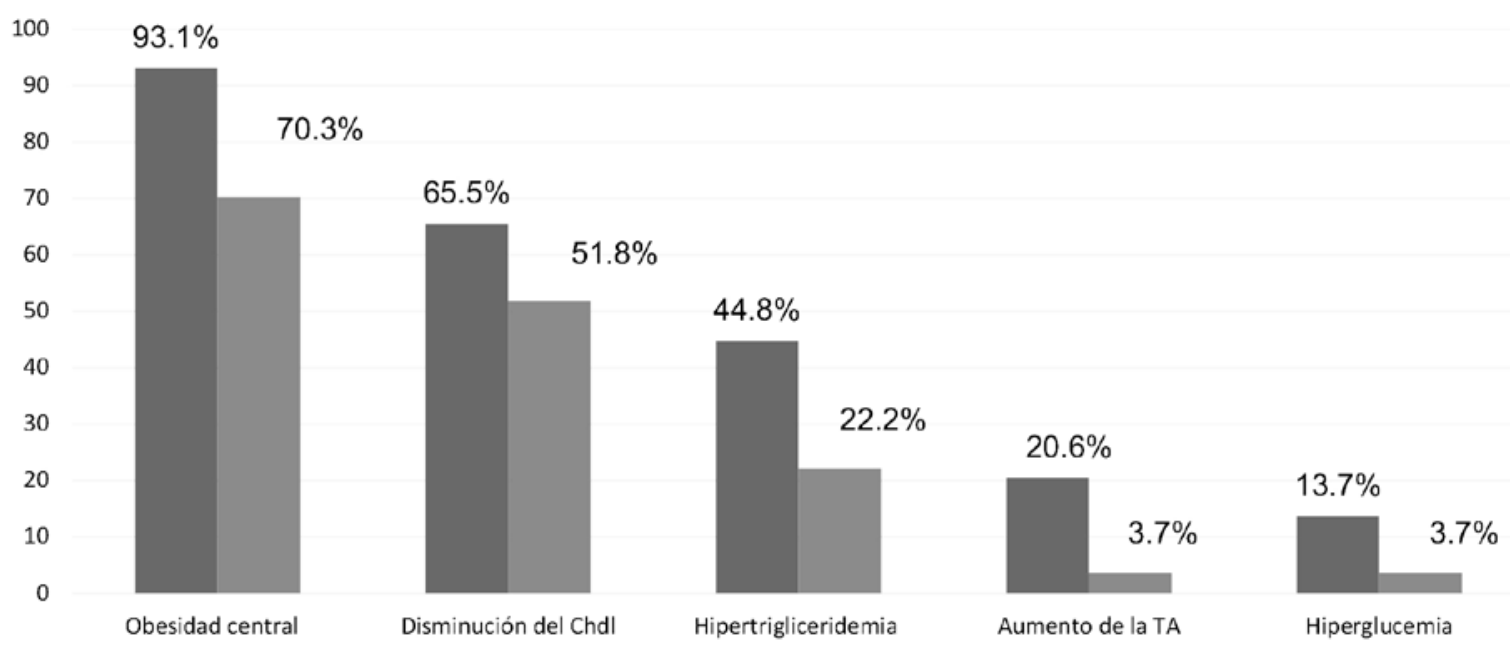

Figura 2. Componentes del síndrome metabólico de acuerdo al sexo de los participantes. cHDL: colesterol lipoproteínas de baja densidad; TA: tensión arterial.

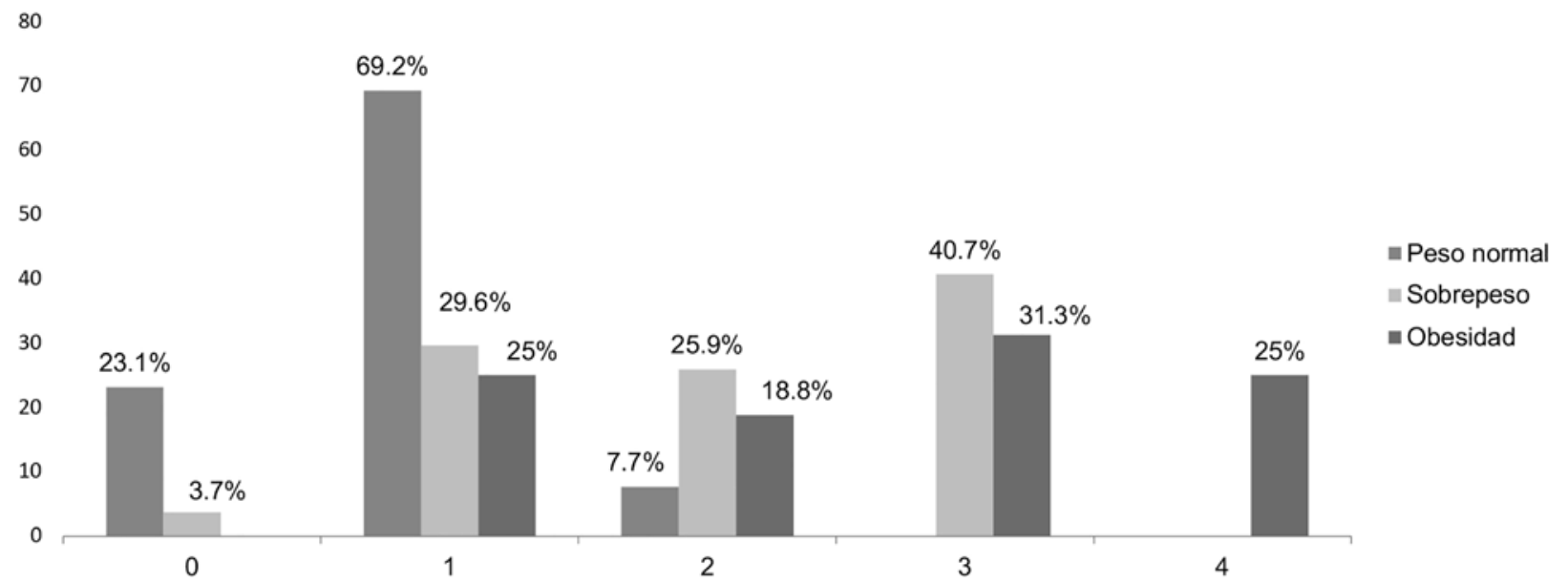

Figura 3. Número de alteraciones metabólicas según el índice de masa corporal de los participantes.

mayor a la descrita en profesionales de la salud de hospitales mexicanos. En Colombia, Forero, et al. reportaron una prevalencia de SM del $19.9 \%$ en una muestra de adultos de entre 20 y 60 años de edad². Por otra parte, Quiroz, et al. encontraron una prevalencia de SM del $26.6 \%$ en profesionales de la salud del Hospital Regional de Xalapa; cabe resaltar que en dicho estudio también se aplicaron los criterios de la IDF ${ }^{14}$.

Respecto a la prevalencia de SM de acuerdo con el sexo y la edad de los participantes, se coincide con Palacios, et al. ${ }^{15,16}$ al encontrar una prevalencia mayor en los hombres y en los participantes mayores de 40 años. Como ya se ha reportado en estudios previos, la prevalencia del SM se relaciona con la edad de los participantes ${ }^{17}$. Cuando se analizaron los criterios de la IDF para el diagnóstico de SM, los componentes que se presentaron con mayor frecuencia fueron la obesidad central y la disminución del cHDL, coincidiendo con estudios previos en profesionales de la salud del IMSS $^{16,18}$. De acuerdo con Ezquerra, et al. (2008), la obesidad central es una de las características principales del $\mathrm{SM}^{19}$, dado que el aumento de la adiposidad, particularmente en depósitos viscerales, conduce al aumento del flujo de ácidos grasos libres y a la inhibición de la acción de la insulina ${ }^{20,21}$.

De acuerdo a los resultados obtenidos de la cuantificación de los macronutrientes y kilocalorías consumidos en la dieta de los residentes médicos, se encontró mayor consumo de $\mathrm{HCO}$, proteínas y lípidos en los residentes con SM comparados con aquellos sin SM. Lo anterior podría deberse a diferentes factores, como las jornadas excesivas de trabajo o el desgaste físico y 
mental, los cuales no permiten que los residentes lleven una dieta adecuada y equilibrada, además del sedentarismo y la escasa actividad física.

Arpa, et al. enfatizan que el conocimiento de los hábitos alimentarios de una población contribuirá a identificar factores de riesgo modificables que impactan en la prevalencia de complicaciones cardiovasculares como el SM ${ }^{22}$. Además, la evidencia científica ha demostrado que los alimentos que se consumen en la dieta y el estilo de vida influyen en las concentraciones de lípidos en forma favorable o desfavorable. De acuerdo con García, et al., la dieta elevada en grasas e hidratos de carbono (> $60 \%$ de las calorías totales) favorece el desarrollo de dislipidemias ${ }^{23}$.

Coincidiendo con los resultados anteriores, Lee, et al. encontraron asociación entre la prevalencia de SM y el consumo de lípidos y ácidos grasos saturados ${ }^{24}$. Es por ello por lo que, además de reducir la ingesta calórica, se deben seguir las recomendaciones nutricionales de los principales grupos de alimentos para fomentar hábitos alimenticios saludables ${ }^{25,26}$ y prevenir las complicaciones metabólicas que deterioran la calidad de vida de los residentes médicos. Por su parte, Orozco, et al. sugieren reorientar los programas de promoción de la salud en las unidades médicas para mejorar el perfil epidemiológico de los profesionales ${ }^{6}$.

Finalmente, el presente estudio permitió conocer la prevalencia de SM en médicos residentes del IMSS, San Luis Potosí, S.L.P., en el ciclo 2014-2015, la cual fue mayor que la prevalencia nacional descrita en población general y en personal de la salud. Respecto a los componentes del SM, los criterios que se presentaron con mayor frecuencia fueron la obesidad central y la disminución del cHDL. Estos se presentaron con mayor frecuencia en los hombres, colocándolos en mayor riesgo de presentar enfermedad cardiovascular aterosclerótica a edades tempranas. En contraste, el aumento de la presión arterial y la hiperglucemia fueron los criterios menos frecuentes. En lo que corresponde a los hábitos alimentarios, se encontró mayor consumo de HCO, proteínas y lípidos en los residentes con SM. Se sugiere que futuras investigaciones se enfoquen en desarrollar e implementar programas multidisciplinarios que favorezcan la modificación del estilo de vida y el fomento de hábitos alimentarios saludables en los médicos residentes.

\section{BIBLIOGRAFÍA}

1. Aguilar-Salinas CA, Rojas R, Gómez-Pérez FJ, Valles V, Ríos-Torres JM, Franco $A$, et al. High prevalence of metabolic syndrome in Mexico. Arch Med Res. 2004;35(1):76-81.

2. Forero Y, Morales GE, Benítez E. Comparación de dos metodologías utilizadas para la determinación del Síndrome Metabólico en población adulta. Biomédica. 2013;33:233-40.

3. Tanchoco CC, Cruz AJ, Duante C, Litonjua A. Prevalence of metabolic syndrome among Filipino adults aged 20 years and over. Asia Pacific J Clin Nutr. 2003;12(3):271-6.

4. Encuesta Nacional de Salud y Nutrición de Medio Camino 2016 [Internet] México: Instituto Nacional de Salud Pública; 2016. Disponible en: http://www. epidemiologia.salud.gob.mx/doctos/encuestas/resultados/ENSANUT.pdf

5. Escasany M, Tumminello MJ, González GA. Síndrome metabólico en personal de enfermería. Rev Esp Nutr Humana Diet. 2012;16(3):89-93.

6. Orozco-González CN, Cortés-Sanabria L, Viera-Franco JJ, Ramírez-Márquez JJ, Cueto-Manzano AM. Prevalencia de factores de riesgo cardiovascular en una población de trabajadores de la salud. Rev Med Inst Mex Seguro Soc. 2016:54(5):594-601.

7. International Society for the Advancement ok Kinanthropometry (ISAK). Estándares Internacionales para Mediciones Antropométricas. ISAK 5ta edición. Australia; 2005.

8. Norma Oficial Mexicana NOM-030-SSA2-2009, para la prevención, detección, diagnóstico, tratamiento y control de la hipertensión arterial sistémica. DOF 18-09-2009.

9. Organización Mundial de la Salud. Manual de bioseguridad en el laboratorio. 3. a ed. Ginebra: Organización Mundial de la Salud; 2005.

10. Zimmet P, Alberti KG, Serrano M. Una nueva definición mundial del síndrome metabólico propuesta por la Federación Internacional de Diabetes: fundamento y resultados. Rev Esp Cardiol. 2005;58(12):1371-6.

11. Montes de Oca E, Loria-Castellanos J, Chavarría-Islas RA. Prevalencia y factores de riesgo para el desarrollo del síndrome metabólico en personal médico de un servicio de urgencias. Rev Cub Med Int Emerg. 2008;7(3):1260-72.

12. Secretaría de Salud. Reglamento de la Ley General de Salud en materia de investigación para la salud. DOF 2-04-2014.

13. Declaración de Helsinki de la Asociación Médica Mundial - Principios éticos para las investigaciones médicas en seres humanos [Internet]. Asociación Médica Mundial; 2014 [consultado en junio de 2014]. Disponible en: http:// www.wma.net/es/30publications/10policies/b3/

14. Quiroz-Cortés MC, Mendoza-González MF, Zendejas-Reyes V. Prevalencia del síndrome metabólico en trabajadores de la salud. México: Facultad de Medicina, Universidad Veracruzana; 2014.

15. Palacios-Rodríguez RG, Munguía-Miranda C, Ávila-Leyva A. Sobrepeso y obesidad en personal de salud de una Unidad de Medicina Familiar. Rev Med Inst Mex Seguro Soc. 2006;44(5):449-53.

16. Palacios-Rodríguez RG, Paulín-Villalpando P, López-Carmona JM, Valerio-Acosta MML, Cabrera-Gaytán DA. Síndrome metabólico en personal de salud de una Unidad de Medicina Familiar. Rev Med Inst Mex Seguro Soc. 2010;48(3):297-302.

17. Damaso B, Loza C, Menacho L. Prevalencia del síndrome metabólico en trabajadores activos en la Red Asistencial de EsSalud en Huánuco, 2007. Rev Med Hered. 2011;22(2):54-62.

18. Padierna-Luna JL, Ochoa-Rosas FS, Jaramillo-Villalobos B. Prevalencia de síndrome metabólico en trabajadores del IMSS. Rev Med Inst Mex Seguro Soc. 2007;45(6):593-9.

19. Ezquerra EA, Castellano JM, Alegría A. Obesidad, síndrome metabólico y diabetes: implicaciones cardiovasculares y actuación terapéutica. Rev Esp Cardiol. 2008;61:752-64

20. López-Albornoz R, Pérez-Rodrigo I. Nutrición y síndrome metabólico. Nutr Clin Diet Hosp. 2012;32(3):92-7.

21. Furukawa S, Fujita T, Shimabukuro M, Iwaki M, Yamada Y, Nakajima Y, et al. Increased oxidative stress in obesity and its impact on metabolic syndrome. J Clin Invest. 2004;114(12):1752-61.

22. Arpa-Gámez AM, González-Sotolongo 0, Vega-Fernández C. Hábitos y estilos de vida asociados al síndrome metabólico. Rev Cubana Med Milit. 2010;39(1):16-26.

23. García-García MR, Panduro A, Martínez-López E. Implicación de los factores dietéticos en el desarrollo de la dislipidemia y su tratamiento nutricional. Rev Mex Endocrinol Metab Nutr. 2014;1:55-62.

24. Lee KW, Cho Y, Jo G, Park YK, Shin MJ. Association of dietary intakes of total and subtypes of fat substituted for carbohydrate with metabolic syndrome in Koreans. Endocr J. 2016;63(11):991-9.

25. Pattyn N, Cornelissen VA, Toghi Eshghi S, Vanhees L. The effect of exercise on the cardiovascular risk factors constituting the Metabolic Syndrome. A meta-analysis of controlled trials. Sports Med. 2013;43:121-33.

26. Tanchoco CC, Cruz AJ, Duante C, Litonjua A. Prevalence of metabolic syndrome among Filipino adults aged 20 years and over. Asia Pacific J Clin Nutr. 2003;12(3):271-6. 